\title{
Comparing Models of Frailty: The Health and Retirement Study
}

\author{
Christine T. Cigolle, MD, MPH, ${ }^{* \dagger}$ Mary Beth Ofstedal, PhD, ${ }^{\ddagger}$ Zhiyi Tian, MA, MS, ${ }^{\mathcal{S}}$ and \\ Caroline S. Blaum, MD, $M S^{\dagger \mathcal{S}}$
}

OBJECTIVES: To operationalize and compare three models of frailty, each representing a distinct theoretical view of frailty: as deficiencies in function (Functional Domains model), as an index of health burden (Burden model), and as a biological syndrome (Biologic Syndrome model).

DESIGN: Cross-sectional analysis.

SETTING: 2004 wave of the Health and Retirement Study, a nationally representative, longitudinal health interview survey.

PARTICIPANTS: Adults aged 65 and older $(\mathrm{N}=11,113)$ living in the community and in nursing homes in the United States.

MEASUREMENTS: The outcome measure was the presence of frailty, as defined according to each frailty model. Covariates included chronic diseases and sociodemographic characteristics.

RESULTS: Almost one-third (30.2\%) of respondents were frail according to at least one model; $3.1 \%$ were frail according to all three models. The Functional Domains model showed the least overlap with the other models. In contrast, $76.1 \%$ of those classified as frail according to the Biologic Syndrome model and $72.1 \%$ of those according to the Burden model were also frail according to at least one other model. Older adults identified as frail according to the different models differed in sociodemographic and chronic disease characteristics. For example, the Biologic Syndrome model demonstrated substantial associations with older age (adjusted odds ratio $(\mathrm{OR})=10.6,95 \%$ confidence interval $(\mathrm{CI})=6.1-18.5)$, female sex $(\mathrm{OR}=1.7,95 \% \mathrm{CI}=1.2-2.5)$, and African-American ethnicity $(\mathrm{OR}=2.1, \% \mathrm{CI}=1.0-4.4)$. CONCLUSION: Different models of frailty, based on different theoretical constructs, capture different groups of older adults. The different models may represent different frailty pathways or trajectories to adverse outcomes such as disability and death. J Am Geriatr Soc 57:830-839, 2009.

Key words: frailty; disability; chronic disease

From the *Department of Family Medicine, ${ }^{\dagger}$ Institute for Social Research, and ${ }^{\S}$ Department of Internal Medicine, University of Michigan, Ann Arbor, Michigan; and ${ }^{\dagger}$ Geriatric Research, Education and Clinical Center, Ann Arbor Veterans Affairs Healthcare System, Ann Arbor, Michigan.

Address correspondence to Christine T. Cigolle, University of Michigan 300 N. Ingalls, Room 919, Ann Arbor, MI 48109. E-mail: ccigolle@umich.edu

DOI: $10.1111 / \mathrm{j} .1532-5415.2009 .02225 . \mathrm{x}$
$F_{\mathrm{u} s}^{\mathrm{r}}$ railty has been an imprecise and variously defined term used to indicate that heterogeneous group of older adults who undergo decline and are especially vulnerable. ${ }^{1-4}$ Models have arisen to define and operationalize frailty in a more specific and standardized manner. These models derive from distinctive theoretical views of how frailty develops and manifests itself in older adults. Research comparing the models is limited, yet how frailty is understood and modeled affects aging research, for example, where frailty fits into the constructs of morbidity, disability, and death and how frailty can improve understanding of prognosis and the effect of illness on patients and families. From a clinical standpoint, how frailty is understood affects efforts to compress morbidity, enhance function, and delay death.

The present study investigated three models, each representing a distinctive perspective of frailty. It compared the models and the older adults that the models identify as frail. Several related models were not included in this study because they are more focused in scope (e.g., sarcopenia, ${ }^{5}$ failure to thrive ${ }^{6}$ ) or are research models with less direct applicability to clinical settings and population-based research (e.g., allostatic load, which includes multiple serum and urine biomarker measures ${ }^{7}$ ).

Strawbridge and colleagues conceived of frailty as "a grouping of problems and losses of capability which make the individual more vulnerable to environmental challenge" and proposed an early frailty model based on deficiencies in four domains of functioning (physical, nutritive, cognitive, and sensory) (Table 1). ${ }^{8}$ They developed this Functional Domains model of frailty in the Alameda County Study (ACS). ${ }^{8}$ The model was the first to combine deficits across these domains into a single measure.

Rockwood and colleagues developed a frailty index (FI) as a measure of deficit accumulation, that is, a measure of the cumulative burden of, for example, symptoms, diseases, conditions, and disability (Table 2). ${ }^{9}$ They developed this Burden model of frailty in the Canadian Study of Health and Aging. ${ }^{10,11}$ In contrast to the Functional Domains model, the items that comprise the FI include but are not limited to conditions that are geriatric in nature.

Fried and colleagues postulated frailty to be a "biologic syndrome of decreased reserve and resistance to stressors, resulting from cumulative declines across multiple 
Table 1. Measures Used to Operationalize Functional Domains Model: Frailty as a Syndrome of Deficiencies in Domains of Functioning

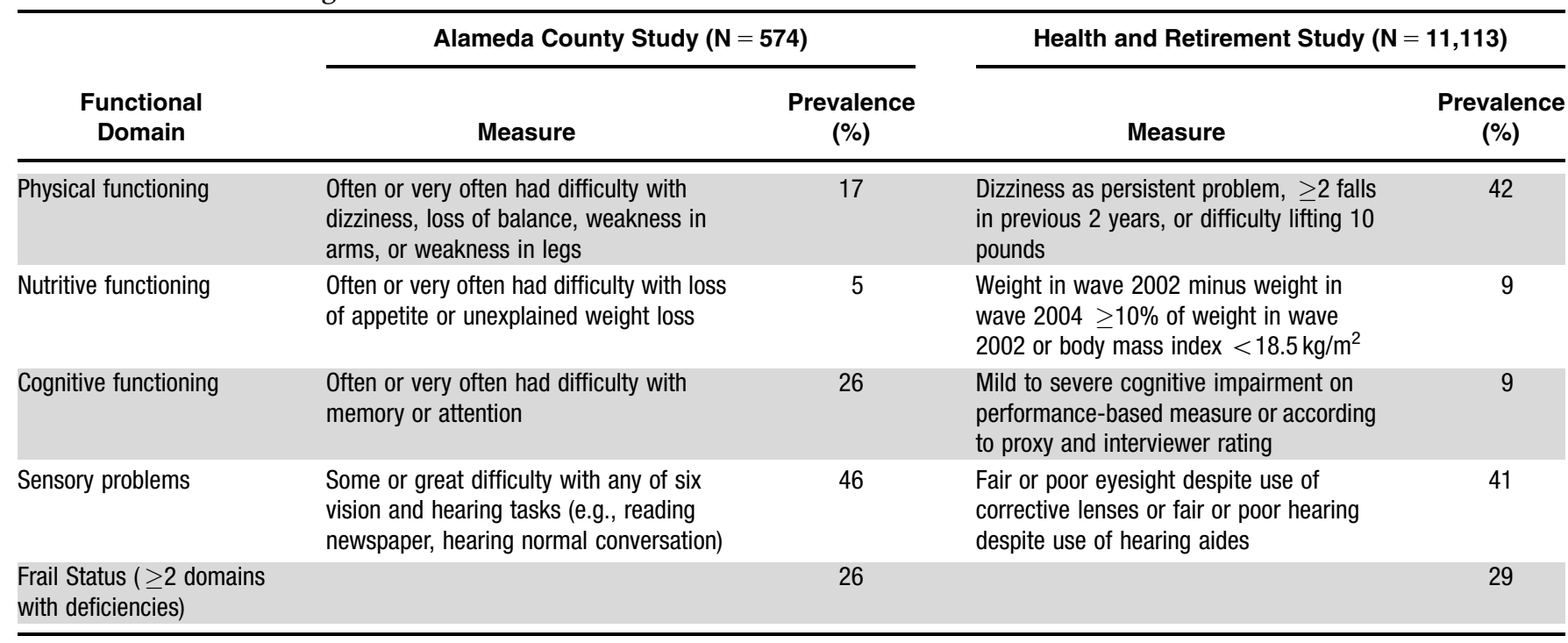

Weighted percentages were derived using Health and Retirement Study respondent population weights to adjust for differential probability of selection into the sample and differential nonresponse.

Adults aged $\geq 65$.

physiologic systems," and defined a frailty phenotype in terms of five components present in a hypothesized cycle of frailty (Table 3). ${ }^{12,13}$ Two components are defined in terms of physical performance measures. They developed this Biologic Syndrome model of frailty in the Cardiovascular Health Study (CHS). ${ }^{14}$ The model was later replicated in the Women's Health and Aging Studies (WHAS) in women aged 70 to $79 .{ }^{15}$

The goal of the current research was to operationalize and compare the three frailty models in a nationally representative population sample, the 2004 wave of the Health and Retirement Study (HRS). The models were analyzed in two steps. First, each model was operationalized in the HRS population and compared with the original model in the original study population. Second, the three models were compared with each other. It was hypothesized that the frailty models, having different theoretical foundations, would differ in identifying older adults as frail. It was further hypothesized that sociodemographic and clinical differences would exist between the populations identified as frail according to the three models.

\section{METHODS}

Data

The data used in this study were from the 2004 wave of the HRS, a population-based longitudinal health interview survey of a cohort of adults aged 51 and older in the United States. ${ }^{16,17}$ Sponsored by the National Institute on Aging and performed by the Institute for Social Research at the University of Michigan, the HRS is designed to study health transitions in older adults. The HRS conducts interviews with respondents and their spouses. Respondents include adults living in the community and those residing in longstay nursing facilities.

The Health Sciences Institutional Review Board at the University of Michigan approved the HRS. The data used for this analysis are publicly available and contain no unique identifiers, thus ensuring respondent anonymity.

\section{Study Population}

Of the 20,129 respondents interviewed in the 2004 wave core survey, 11,113 were aged 65 and older (Figure 1). When the eligible respondent was unable to be interviewed, often because of medical or cognitive problems, a proxy $(\mathrm{n}=1,226)$, frequently the spouse $(\mathrm{n}=649)$, was enlisted to answer questions for that respondent. The 2004 wave included physical performance measures on a subsample of community-dwelling nonproxied respondents $(\mathrm{n}=3,274)$; of these, 2,111 were aged 65 and older.

\section{Variables and Their Measurement Frailty}

Data from the core survey and from the physical performance measures subsample (for the Biologic Syndrome model) were used to operationalize the three frailty models in the HRS (Tables 1, 2, and 3).

Functional Domains Model. The original model in the ACS consisted of 16 self-report items grouped into four domains of functioning. ${ }^{8}$ Subjects were classified as frail if they had difficulties in two or more domains. The physical functioning domain in the ACS included dizziness, loss of balance, weakness in the arms, and weakness in the legs. Physical functioning was defined in the HRS as dizziness as a persistent problem, two or more falls in the previous 2 years, or difficulty lifting 10 pounds. The nutritive functioning domain in the ACS included loss of appetite and unexplained weight loss. Nutritive functioning was defined in the HRS as $10 \%$ or greater weight loss in the previous 2 years (derived from comparison of self-reported weight in 2004 and 2002) or a body mass index (BMI) less than $18.5 \mathrm{~kg} / \mathrm{m}^{2}$ (derived from self-reported height and weight). The cognitive functioning domain in the ACS included 


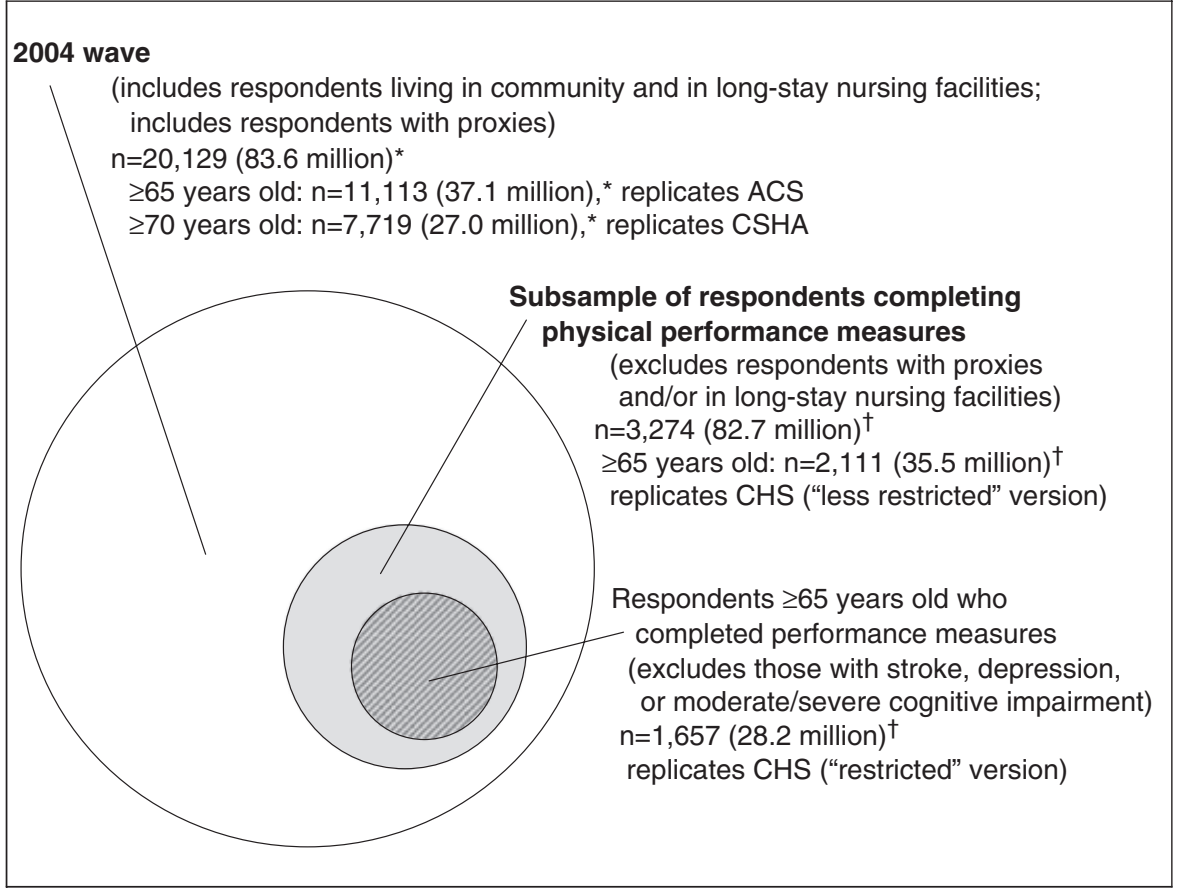

Figure 1. Diagram depicting Health and Retirement Study (HRS) 2004 wave population sample and physical performance measures population subsample. Weighted percentages were derived using HRS respondent population weights to adjust for differential probability of selection into the sample and differential nonresponse. *National population estimates after weighting, using respondent weights for entire 2004 wave sample. ${ }^{\dagger}$ National population estimates after weighting, using respondent weights for 2004 wave physical performance measures sub-sample. ACS = Alameda County Study; CSHA = Canadian Study of Health and Aging; CHS = Cardiovascular Health Study.

memory and attention difficulties. Cognitive functioning was defined in the HRS using a performance-based measure to assess the degree of cognitive impairment (see below). The sensory functioning domain in the ACS included vision and hearing difficulties in different situations. Sensory functioning was defined in the HRS as fair or poor vision or hearing despite the use of corrective lenses or hearing aides.

Burden Model. The FI in the CHAS comprised 70 measures that may be generally categorized as diseases, neurological conditions, or impairments in cognition, mood, mobility, or function. ${ }^{9}$ Thirty-eight of the measures were able to be replicated in the HRS, including at least $60 \%$ of the measures in each general category (except neurological conditions), thus achieving a broad representation of the accumulated deficits that the FI was intended to signify. (The individual measures for each respondent were mapped to an interval scale (from 0 to 1 ) to produce the composite FI, scaled from 0 to 1 , for that respondent.) The FI, as originally developed, conceptualizes frailty as a continuum, but researchers have used FI values of 0.2 and of 0.25 as cutpoints to distinguish frail from not frail..$^{9}$ The current study used a FI of 0.2 or above to classify respondents as frail. (Analyses were also performed with a FI of 0.25 or above (data not shown).)

Biologic Syndrome Model. The original model was specified using five frailty criteria. ${ }^{13}$ Subjects were classified as frail if they met three or more criteria. Weight loss was defined in the CHS as the self-reported unintentional loss of 10 or more pounds in the previous year. Weight loss was defined in the HRS as $10 \%$ or greater weight loss in the previous 2 years or a BMI less than $18.5 \mathrm{~kg} / \mathrm{m}^{2}$. The same Center for Epidemiologic Studies Depression Scale (CES-
D) ${ }^{18}$ measures used in the CHS to define exhaustion were also used in the HRS; respondents answered yes or no to whether they had experienced either CES-D item for much of the time during the previous week. Low activity was defined in the CHS in terms of kilocalories expended per week, based on responses to selected items from the Minnesota Leisure Time Activity questionnaire. ${ }^{15,19,20}$ Three questions in the HRS asked respondents about their frequency of mild, moderate, and vigorous physical activities. An activity scale weighted according to the intensity and the frequency of these activities was constructed. ${ }^{21}$ Low activity was defined in the HRS as the lowest $20 \%$ for each sex. Slowness was defined in the HRS in terms of usual-pace walking speed measured over 8 feet (vs 15 feet in the CHS), using the same cutpoints as in the CHS (after adjusting for distance). Weakness was defined in the HRS by assessing grip strength, using the same cutpoints as in the CHS. Respondents unable to complete the respective physical performance tests were included as slow and as weak.

\section{Chronic Diseases}

The following chronic diseases were included in the analyses: hypertension, heart disease, chronic lung disease, diabetes mellitus, cancer, musculoskeletal conditions, and psychiatric problems. Each disease was limited to its active or severe form (e.g., receiving treatment for the disease). ${ }^{22,23}$

Stroke and depression were exclusion criteria for some analyses (see below for further explanation). Respondents with stroke were defined as those reporting stroke who required medication for the stroke (or its complications) or who had remaining problems from the stroke. Respondents 
with depression were defined as those with a positive response to four (of eight) CES-D items.

\section{Cognitive Impairment}

Cognitive impairment (mild to severe) is a component in the Functional Domains model and in the Burden model. (In addition to stroke and depression, moderate to severe cognitive impairment was a third exclusion criterion for some analyses.) Cognitive status was assessed using a performance-based measure, a modified version of the Telephone Interview for Cognitive Status (TICS), a validated cognitive screening instrument patterned on the Mini-Mental State Examination ${ }^{24}$ and specifically designed for population-based studies. ${ }^{25-27}$ Moderate to severe impairment was defined as a score of 7 or less on the 35 -point cognitive scale and mild impairment as a score of 8 to 10 . For respondents unable to complete the interview, a proxy respondent was asked to assess the respondent's memory. The presence and degree of cognitive impairment for these respondents were determined by combining the proxy's assessment of the respondent's memory with the interviewer's assessment of the respondent's cognitive function. ${ }^{27}$

\section{Sociodemographic Characteristics}

Sociodemographic variables included age, sex, race (Caucasian, African American, Hispanic), marital status, educational attainment, net worth (total household assets minus current debt), ${ }^{16}$ and living arrangement (lives with others, alone, or in a long-stay nursing facility).

\section{Statistical Analysis}

To adjust for the complex sample design of the HRS, the differential probability of selection, and nonresponse, all analyses were weighted and adjusted using the statistical package Stata (Release 9.0, StataCorp., College Station, TX). The 2004 wave sample and the physical performance measures subsample (Figure 1) had different sets of respondent weights, each set specific to each sample. As a result, for both the 2004 wave sample and the subsample, the study was able to take advantage of the nationally representative data to produce national population estimates.

The first set of analyses (Tables 1, 2, and 3) estimated the prevalence of frailty for each frailty model, as defined in the HRS, and compared it with that of the original model, as developed in the original study population. For each frailty model, the HRS population sample was selected so as to replicate, as closely as possible, each original study population and its eligibility criteria (Figure 1). To replicate the ACS, ${ }^{28}$ the Functional Domains model was developed in the HRS 2004 wave sample of adults aged 65 and older. The sample consisted of 11,113 respondents, representing 37.1 million adults aged 65 and older in the United States in 2004. To replicate the CHAS, ${ }^{10,11}$ the Burden model was developed in the HRS 2004 wave sample of adults aged 70 and older. The sample consisted of 7,719 respondents, representing 27.0 million adults aged 70 and older nationally. To replicate the CHS,${ }^{14}$ the Biologic Syndrome model was developed in the sample of HRS respondents aged 65 and older who completed the performance measures and did not have stroke, depression, or moderate to severe cognitive impairment (single diseases or conditions that could by themselves result in frailty characteristics). ${ }^{13}$ The resulting sample consisted of 1,657 respondents, representing 28.2 million adults aged 65 and older nationally.

The second set of analyses (Figure 2, Tables 4 and 5) compares the three frailty models with one another in the same HRS population sample. For this comparison, the more "restricted" population sample (the sample that replicated the CHS) was chosen: respondents were aged 65 and older; completed the performance measures; and did not have stroke, depression, or moderate to severe cognitive impairment $(\mathrm{n}=1,657)$.

The third set of analyses (data not shown) also compares the three frailty models with one another in the same population sample, the sample of HRS respondents aged 65 and older who completed the performance measures $(n=2,111)$. For this "less restricted" sample, the exclusion criteria of stroke, depression, and moderate to severe cognitive impairment were omitted.

Standard descriptive methods were used to estimate prevalences, determine confidence intervals, and make comparisons between frail groups. Logistic regression models were used to examine the multivariate association between frailty and sociodemographic characteristic and chronic disease covariates. The dependent variable in these models was frailty, as defined according to each frailty model.

\section{RESULTS}

The first set of analyses (Tables 1, 2, and 3) compared each frailty model, as developed in the HRS, with the original model, as developed in the original study population. For example, the Biologic Syndrome model in the HRS was compared with the Biologic Syndrome model in the CHS. For each model, the prevalence of frailty and of each frailty criterion in the HRS population was compared with that in the original study populations. In general, prevalences of frailty in the HRS were consistent with those in the original study populations: for example, Functional Domains model, HRS 29\%, ACS 26\%, and Biologic Syndrome model, HRS $11 \%$, CHS $7 \%$. The greatest discrepancies were found for the individual frailty criteria in the Functional Domains model.

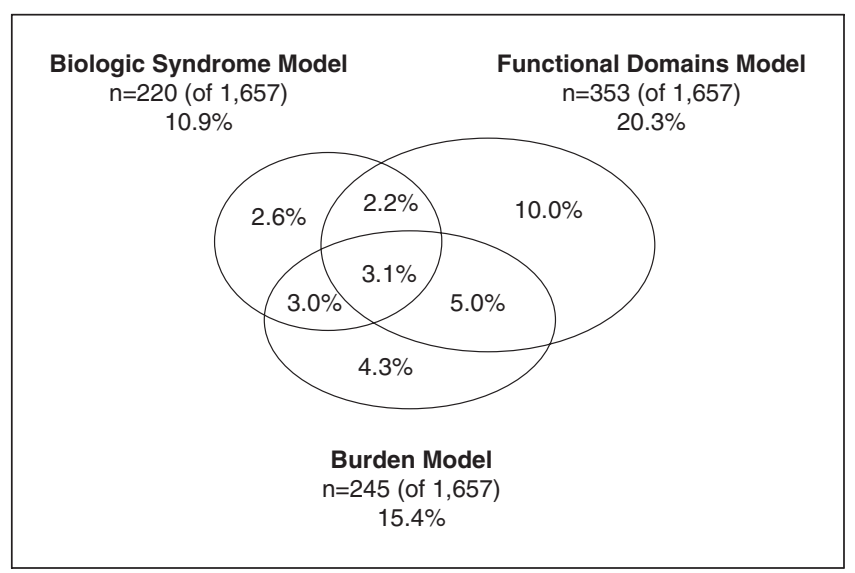

Figure 2. Venn diagram depicting how the models differ in identifying respondents as frail. Weighted percentages were derived using Health and Retirement Study respondent population weights to adjust for differential probability of selection into the sample and differential nonresponse. 
Table 2. Measures Used to Operationalize Burden Model: Frailty as an Index of Deficit Accumulation

\begin{tabular}{|c|c|c|c|c|}
\hline Frailty Index & $\begin{array}{l}\text { Canadian Study of } \\
\text { Health and Aging } \\
\quad(\mathrm{N}=2,305)\end{array}$ & $\begin{array}{c}\text { Prevalence } \\
\text { (\%) }\end{array}$ & $\begin{array}{c}\text { Health and } \\
\text { Retirement } \\
\text { Study } \\
(\mathrm{N}=7,719)\end{array}$ & $\begin{array}{c}\text { Prevalence } \\
\text { (\%) }\end{array}$ \\
\hline $\begin{array}{l}\text { Cumulative burden scale comprising diseases, } \\
\text { neurological conditions, and impairments in cognition, } \\
\text { mood, mobility, and function measures }\end{array}$ & $\begin{array}{l}\text { 70-item scale; Frailty } \\
\text { Index } \geq 0.25\end{array}$ & Not available & $\begin{array}{l}\text { 38-item scale; Frailty } \\
\text { Index } \geq 0.2\end{array}$ & 32 \\
\hline
\end{tabular}

Weighted percentages were derived using Health and Retirement Study respondent population weights to adjust for differential probability of selection into the sample and differential nonresponse.

Adults aged $\geq 70$.

The prevalence of frailty and of each frailty criterion for the Biologic Syndrome model in the HRS was also compared with that in the CHS and in the WHAS for the subgroup of women aged 70 to $79(\mathrm{n}=367$, data not shown). The prevalence of frailty for women in this age group in the HRS $(10.5 \%)$ was consistent with that for the CHS $(7.3 \%)$ and the WHAS $(12.7 \%)$.

The next set of analyses (Figure 2, Tables 4 and 5) compared the three frailty models with one another in the same population sample, the "restricted" HRS subsample of 1,657 respondents. The frailty models differed substantially in how they classified respondents as frail. The Venn diagram in Figure 2 illustrates how the populations identified as frail according to each model overlapped. Overall, $30.2 \%$ of respondents were frail according to at least one model and $3.1 \%$ according to all three models. The Functional Domains model showed the least overlap with the other models. In contrast, $76.1 \%$ of those classified as frail according to the Biologic Syndrome model and $72.1 \%$ of those according to the Burden model were also frail according to at least one other model (Table 4).

Table 4 shows the weighted distributions for key demographic, socioeconomic, and health covariates for the study population $(\mathrm{n}=1,657)$ (column 1 ) and for the respondents classified as frail according to each frailty model (columns 2-4). Respondents classified as frail generally were older; were more likely to be female, from a minority ethnic group, and unmarried; had less education and a lower net worth; and were more likely to live alone than those not identified as frail. However, notable differences were found between the models. For example, frailty according to the Biologic Syndrome model had substantially greater prevalence in the oldest-old, women, and African Americans. These findings were present but less marked in the Functional Domains and Burden groups.

\begin{tabular}{|c|c|c|c|c|}
\hline \multirow[b]{2}{*}{$\begin{array}{l}\text { Frailty-Defining } \\
\text { Criterion }\end{array}$} & \multicolumn{2}{|c|}{ Cardiovascular Health Study $(\mathrm{N}=5,317)^{*}$} & \multicolumn{2}{|l|}{ Health and Retirement Study $(\mathrm{N}=1,657)^{\dagger}$} \\
\hline & Measure & $\begin{array}{l}\text { Prevalence } \\
\text { (\%) }\end{array}$ & Measure & $\begin{array}{l}\text { Prevalence } \\
\quad(\%)\end{array}$ \\
\hline Weight loss & Lost $>10$ pounds unintentionally in last year & 6 & $\begin{array}{l}\text { Weight in wave } 2002 \text { minus weight in wave } 2004 \\
\geq 10 \% \text { of weight in wave } 2002 \text { or BMl }<18.5 \mathrm{~kg} / \mathrm{m}^{2}\end{array}$ & 7 \\
\hline $\begin{array}{l}\text { Low energy } \\
\text { expenditure }\end{array}$ & $\begin{array}{l}\text { Kilocalories of physical expenditure per week, based } \\
\text { on } 18 \text {-item activity scale, }{ }^{\ddagger} \text { lowest quintile (stratified } \\
\text { according to sex) }\end{array}$ & 22 & $\begin{array}{l}\text { Frequency of three intensities of activity, lowest } \\
\text { quintile (stratified according to sex) }\end{array}$ & 20 \\
\hline Slowness & $\begin{array}{l}\text { Time to walk } 15 \mathrm{ft} \text { : Slowest } 20 \% \text { (stratified according } \\
\text { to sex and height) }\end{array}$ & 20 & $\begin{array}{l}\text { Time to walk } 8 \mathrm{ft} \text {, converted to time to walk } 15 \mathrm{ft} \text {. Cutoff } \\
\text { criteria according to sex and height remain the same }\end{array}$ & 30 \\
\hline
\end{tabular}

Weighted percentages were derived using Health and Retirement Study respondent population weights to adjust for differential probability of selection into the sample and differential nonresponse.

* Adults aged $\geq 65$ who completed physical performance measures, excluding those with Parkinson's disease; stroke; Mini-Mental State Examination scores $<18$; or taking carbidopa-levodopa, donepezil hydrochloride, or antidepressants.

${ }^{\dagger}$ Adults aged $\geq 65$ who completed physical performance measures, excluding those with stroke, moderate to severe cognitive impairment, or depression ( $\geq 4$ out of 8 Center for Epidemiologic Studies Depression Scale (CES-D) items).

${ }^{\ddagger}$ Minnesota Leisure Time Activity questionnaire.

$\mathrm{BMI}=$ body mass index. 
Table 4. Characteristics of the Study Population, Overall and According to Frailty Model

Weighted Percentage*

Frailty Models

Characteristic Sample Population $(N=1,657) \quad$ Functional Domains $(n=353) \quad$ Burden $(n=245) \quad$ Biologic Syndrome $(n=220)$

Age

$65-69$

$70-74$

$75-79$

$\geq 80$

$P$-value

Sex

Male

Female

$P$-value

Race

Caucasian

African American

Hispanic

$P$-value

Marital status

Married

Unmarried

$P$-value

Years of education

$<12$
12
$>12$
$P$-value

Net worth, \$

$\leq 40,000$

40,001-155,000

$155,001-420,000$

$>420,000$

$P$-value

Living arrangement

With others

Alone

$P$-value

Chronic diseases

Hypertension

$P$-value

Heart disease

$P$-value

Lung disease

$P$-value

Diabetes mellitus

$P$-value

Cancer

$P$-value

Musculoskeletal

$P$-value

Psychiatric

$P$-value

Number of chronic diseases

$\begin{array}{ll}31.9 & 22.5 \\ 24.0 & 19.2 \\ 17.1 & 17.0 \\ 27.0 & 41.4 \\ & <.001\end{array}$

21.7

10.1

23.0

15.4

13.1

39.9

12.6

$<.001$

64.2

$<.001$

$44.5 \quad 36.3$

63.7

32.3

28.5

55.5

.004

67.7

71.5

.002

$<.001$

$\begin{array}{rr}88.1 & 83.5\end{array}$

7.4

83.5
11.8

85.9

78.2

4.5

4.7

10.1

16.7

.003

3.9

5.1

$<.001$

64.4

35.6

54.5

45.5

.002

52.8

43.3

47.2

56.7

22.2

36.7

41.1

28.4

38.1

33.5

.007

.003

$<.001$

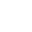

22.0

31.3

21.7

25.1

$<.001$

73.6

26.4

68.6

31.4

.03

32.6

34.9

32.6

.003

35.1

35.3

29.6

$<.001$

16.3
21.5
27.3
34.8

27.6

32.9

30.1

20.7

26.3

21.6

21.9

$<.001$

18.9

$<.001$

69.3

30.7

.21

64.5

35.5

.02

53.6

59.5

.12

23.3

37.1

$<.001$

74.1

64.6

$<.001$

.01

49.6

29.9

4.9

9.1

$<.001$

.03

12.9

12.2

.002

15.7

23.3

$<.001$

3.3

3.9

.57

30.2

39.8

4.0

.001

4.1

.94

$<.001$

$<.001$

29.2

26.8

$<.001$

7.3

.003

51.7

$<.001$

9.2

$<.001$

.001

4.7

.38

48.9

$<.001$

5.6

.28 
Table 4. (Contd.)

Weighted Percentage*

Frailty Models

Characteristic Sample Population $(\mathrm{N}=1,657) \quad$ Functional Domains $(\mathrm{n}=353)$

Burden $(n=245) \quad$ Biologic Syndrome $(n=220)$

\begin{tabular}{|c|c|c|c|c|}
\hline$\geq 1$ & 76.7 & 87.4 & 96.8 & 90.9 \\
\hline$P$-value & & $<.001$ & $<.001$ & $<.001$ \\
\hline$\geq 2$ & 41.7 & 61.8 & 83.8 & 69.0 \\
\hline$P$-value & & $<.001$ & $<.001$ & $<.001$ \\
\hline$\geq 3$ & 13.9 & 22.1 & 39.2 & 25.4 \\
\hline$P$-value & & $<.001$ & $<.001$ & $<.001$ \\
\hline \multicolumn{5}{|c|}{ Cognitive impairment } \\
\hline Mild & 1.8 & 7.0 & 5.9 & 4.1 \\
\hline$P$-value & & $<.001$ & $<.001$ & .16 \\
\hline \multicolumn{5}{|c|}{ Number of activity of daily living dependencies } \\
\hline$\geq 1$ & 4.8 & 15.3 & 25.6 & 20.5 \\
\hline$P$-value & & $<.001$ & $<.001$ & $<.001$ \\
\hline$\geq 2$ & 1.7 & 4.8 & 10.6 & 8.3 \\
\hline$P$-value & & .001 & $<.001$ & $<.001$ \\
\hline$\geq 3$ & 0.6 & 1.5 & 3.7 & 2.5 \\
\hline$P$-value & & .05 & $<.001$ & .008 \\
\hline \multicolumn{5}{|c|}{ Number of instrumental activity of daily living dependencies } \\
\hline$\geq 1$ & 7.4 & 20.0 & 31.4 & 24.2 \\
\hline$P$-value & & $<.001$ & $<.001$ & $<.001$ \\
\hline$\geq 2$ & 2.2 & 8.0 & 11.2 & 8.7 \\
\hline$P$-value & & $<.001$ & $<.001$ & $<.001$ \\
\hline$\geq 3$ & 0.5 & 2.4 & 2.8 & 3.5 \\
\hline$P$-value & & $<.001$ & $<.001$ & $<.001$ \\
\hline
\end{tabular}

* Weighted percentages were derived using Health and Retirement Study respondent population weights to adjust for differential probability of selection into the sample and differential nonresponse.

Proportions are related to the columns and not the rows; the columns for each variable (not the rows) add to $100 \%$. For example, of respondents with frailty (Biologic Syndrome model), $28.5 \%$ were male and $71.5 \%$ female.

$P$-value from the ch-square test for association between the indicated variable and frailty.

Table 4 likewise shows the prevalence of chronic diseases, cognitive impairment, and activity of daily living (ADL) and instrumental activity of daily living (IADL) dependency in the study population and in each frail population. For each model, frail respondents had greater prevalence of heart disease, lung disease, diabetes mellitus, and musculoskeletal disorders. Frail respondents also had greater prevalences of chronic diseases and of ADL and IADL dependencies, which was in general most noted for the Burden model.

Table 5 shows the association between frailty (for each frailty model) and sociodemographic characteristics and chronic diseases using multivariate logistic regression models. The Functional Domains and Biologic Syndrome models were investigated in two steps, first including only sociodemographic variables in the respective models (Model 1 and Model 4) and then introducing chronic disease variables (Model 2 and Model 5). In the Biologic Syndrome model, older age, female sex, African-American ethnicity, lower net worth, lung disease, diabetes mellitus, and musculoskeletal conditions were associated with frailty. These associations generally were also found for the Functional Domains model, although with smaller odds ratios. Chronic disease variables make up the Burden model in part; hence, only the association between frailty and demographic characteristics was examined (Model 3). Significant associations were found for older age, female sex, and net worth. For each of the three frailty models, interactions between the independent variables were systematically tested for, but none that seemed meaningful were found.

Finally, the analyses in Figure 2 and Tables 4 and 5 were repeated on the "less restricted" population sample (including respondents with stroke, depression, and dementia, $\mathrm{n}=2,111$, data not shown). As expected, there was substantially greater prevalence of frailty for each model: Functional Domains, 26.1\%, Burden, 24.7\%, and Biologic Syndrome, $15.9 \%$. Nearly $40 \%$ of respondents were frail according to at least one model, and $7.0 \%$ were frail according to all three models. The association between frailty and African-American ethnicity was smaller than in the analyses that excluded these three diseases or conditions. As expected, the association between frailty and heart disease and psychiatric illness increased. The prevalence of mild to severe cognitive impairment for the models was $12.0 \%$ for Functional Domains, $11.0 \%$ for Burden, and $9.7 \%$ for Biologic Syndrome. 
Table 5. Odds Ratios for Association of Sociodemographic Characteristics and Chronic Diseases with Frailty $(\mathrm{N}=1,657)$

Odds Ratio (95\% Confidence Interval)

\begin{tabular}{|c|c|c|c|c|c|}
\hline \multirow[b]{2}{*}{ Characteristic } & \multicolumn{2}{|c|}{ Functional Domains } & \multirow{2}{*}{$\begin{array}{l}\text { Burden } \\
\text { Model 3* }\end{array}$} & \multicolumn{2}{|c|}{ Biologic Syndrome } \\
\hline & Model $1^{*}$ & Model $\mathbf{2}^{\dagger}$ & & Model 4* & Model $5^{\dagger}$ \\
\hline \multicolumn{6}{|l|}{ Age } \\
\hline $70-74$ & $1.1(0.6-2.0)$ & $1.0(0.5-1.8)$ & $1.3(0.8-2.3)$ & $1.8(0.7-4.3)$ & $1.6(0.6-4.0)$ \\
\hline $75-79$ & $1.5(0.9-2.6)$ & $1.4(1.7-2.5)$ & $1.3(0.7-2.6)$ & $2.6(1.1-6.2)$ & $2.4(1.0-5.5)$ \\
\hline$\geq 80$ & $2.6(1.6-4.2)$ & $2.2(1.4-3.7)$ & $2.2(1.4-3.4)$ & $10.2(5.5-18.8)$ & $10.6(6.1-18.5)$ \\
\hline Female & $1.4(1.0-1.9)$ & $1.5(1.0-2.2)$ & $1.7(1.1-2.5)$ & $1.9(1.3-2.7)$ & $1.7(1.2-2.5)$ \\
\hline \multicolumn{6}{|l|}{ Race } \\
\hline African American & $1.7(1.0-2.6)$ & $1.7(1.1-2.7)$ & $0.5(0.2-1.2)$ & $2.4(1.1-5.0)$ & $2.1(1.0-4.4)$ \\
\hline Hispanic & $1.0(0.5-1.7)$ & $1.1(0.6-2.2)$ & $0.9(0.5-1.8)$ & $1.0(0.4-2.1)$ & $1.0(0.4-2.2)$ \\
\hline Married & $1.1(0.8-1.5)$ & $1.0(0.7-1.5)$ & $1.1(0.8-1.6)$ & $1.3(0.8-2.1)$ & $1.3(0.8-2.1)$ \\
\hline \multicolumn{6}{|l|}{ Years of education } \\
\hline$\leq 12$ & $0.9(0.6-1.4)$ & $1.0(0.7-1.4)$ & $0.6(0.4-1.0)$ & $0.8(0.5-1.4)$ & $0.9(0.5-1.5)$ \\
\hline$>12$ & $0.8(0.5-1.1)$ & $0.8(0.5-1.2)$ & $0.7(0.4-1.1)$ & $0.7(0.4-1.2)$ & $0.8(0.4-1.3)$ \\
\hline \multicolumn{6}{|l|}{ Net worth, \$ } \\
\hline $40,001-155,000$ & $1.3(0.8-2.0)$ & $1.5(1.0-2.3)$ & $0.8(0.5-1.3)$ & $0.6(0.4-0.9)$ & $0.7(0.4-1.1)$ \\
\hline $155,001-420,000$ & $0.6(0.4-1.0)$ & $0.8(0.5-1.1)$ & $0.4(0.2-0.7)$ & $0.4(0.2-0.7)$ & $0.5(0.3-0.9)$ \\
\hline$>420,000$ & $0.6(0.4-1.0)$ & $0.8(0.5-1.3)$ & $0.3(0.2-0.6)$ & $0.3(0.2-0.5)$ & $0.4(0.2-0.7)$ \\
\hline \multicolumn{6}{|l|}{ Chronic diseases } \\
\hline Hypertension & & $1.0(0.7-1.5)$ & & & $1.2(0.9-1.8)$ \\
\hline Heart disease & & $2.4(1.7-3.4)$ & & & $1.3(0.8-2.0)$ \\
\hline Lung disease & & $2.4(1.2-4.5)$ & & & $3.1(1.5-6.4)$ \\
\hline Diabetes mellitus & & $1.7(1.2-2.4)$ & & & $2.2(1.2-3.7)$ \\
\hline Cancer & & $1.1(0.5-2.4)$ & & & $1.6(0.5-4.8)$ \\
\hline Musculoskeletal & & $1.6(1.1-2.3)$ & & & $2.2(1.4-3.4)$ \\
\hline Psychiatric & & $1.0(0.5-2.3)$ & & & $1.9(0.8-4.6)$ \\
\hline
\end{tabular}

Weighted percentages were derived using Health and Retirement Study respondent population weights to adjust for differential probability of selection into the sample and differential nonresponse.

* Adjusted for six demographic characteristics.

${ }^{\dagger}$ Adjusted for six demographic characteristics and seven chronic diseases.

\section{DISCUSSION}

This study examined three models of frailty, each derived from a different theoretical view of how frailty develops and manifests itself in older adults. The goal of the research was to operationalize each model in a population-based sample using performance measures and to compare the older adults identified as frail according to each model. To the authors' knowledge, this is the first study to do so using nationally representative data including older men and women across the age spectrum. Confirming the study's hypotheses, the models differed substantially in identifying older adults as frail, and the individuals identified as frail according to the models differed in their sociodemographic and chronic disease characteristics. Thus, different models of frailty, based on different theoretical constructs, captured different groups of older adults.

The extent to which individual measures in the models can be replicated limits attempts to operationalize and compare models of frailty in population-based data sets. The Functional Domains model, ${ }^{8}$ although older and comprising common domains of functioning, has not been developed in other large datasets. The Burden model ${ }^{9}$ has been developed in the National Long-Term Care Survey and in a number of international data sets. ${ }^{29,30}$ Its $70 \mathrm{com}$ posite measures may limit its replication, although its developers have demonstrated that the model does not depend on any specific measures or on any specific number of measures. ${ }^{9}$ The Biologic Syndrome model ${ }^{13}$ has been widely developed, including in the WHAS, ${ }^{15}$ the Women's Health Initiative Observational Study, ${ }^{31}$ and the Study of Osteoporotic Fractures (women only), ${ }^{32,33}$ the Osteoporotic Fractures in Men Study (men only), ${ }^{34}$ and CHAS (men and women). ${ }^{9}$ Its use of physical performance measures limits its replication; different studies have been able to replicate the performance measures to varying extents, sometimes by substituting self-report measures. The extent to which population samples replicate the eligibility and exclusion criteria of the study populations in which the models were originally developed further limits comparison of frailty models.

How frailty is conceptualized and defined has implications for research investigating its place in the disablement process in older adults. ${ }^{35}$ Frailty is theorized to be related to but distinct from disability, and validity is claimed in part for each of the three models investigated here because of its longitudinal association with disability. Frailty has also 
been referred to as a geriatric syndrome or condition and has been theorized to underlie other geriatric syndromes, thus constituting a common risk factor for the other syndromes. ${ }^{36}$ Yet the exact place of frailty in the disablement pathway and in the pathophysiology of geriatric syndromes in older adults is not clear. Conditions related to frailty (e.g., sarcopenia ${ }^{5}$ and allostatic load ${ }^{7}$ ) are similarly being investigated for their roles in older adult disability.

The current study suggests that different models of frailty capture different but overlapping groups of older adults, with estimates for the prevalence of frailty nationally in community-dwelling older adults ranging from approximately 6 million for the Biologic Syndrome model to 9 million for the Functional Domains and Burden models. Furthermore, the models classify as frail older adults with different sociodemographic and chronic disease profiles. For example, the current study's findings replicated those of other researchers showing a greater prevalence of frailty, as defined according to the Biologic Syndrome model, in the oldest old, women, and African Americans. ${ }^{37}$ These sociodemographic associations were less pronounced for the Functional Domains and Burden models.

One challenge for frailty research is to ascertain the extent to which frailty can be a clinically useful concept, that is, assisting with prognosis. ${ }^{35}$ For instance, there are ongoing efforts to identify frail patients clinically, with the aim of interrupting the frailty process leading to disability and death. Also, identification of patients as frail may better stratify them as appropriate or inappropriate for certain interventions, such as surgical procedures or aggressive chemotherapy. Although none of the frailty models is currently feasible for widespread clinical application, research models will influence any consensus on a clinical definition of frailty. One early frailty screening tool includes many of the items making up the three models investigated in this study. ${ }^{38}$ In comparison, as part of the Assessing Care of the Vulnerable Elders initiative, the Vulnerable Elders Survey provides a clinical measure of vulnerability with the goal of identifying older adults at risk for functional decline or death. ${ }^{39}$ Other researchers have taken a further step and developed a prognostic index for mortality specifically for the frail older adult population. ${ }^{40}$

This study has several limitations. First, the HRS is based on self-report data (other than the physical performance and cognitive measures). Most of the individual measures that define each frailty model derive from selfreport data and are limited by the questions included in the HRS survey, so exact replication of each frailty model is not possible. The questions included in the HRS similarly limit the chronic disease covariates; for example, the survey does not have data on Parkinson's disease. Finally, this study is cross-sectional; further research is needed to examine frailty longitudinally.

The chief strength of this research is that it is based on a large, nationally representative survey - the HRS - that includes physical performance measures and a performance-based determination of cognitive ability, in addition to data on sociodemographic characteristics and chronic diseases. Thus, these data enable the operationalization and comparison of different models of frailty in the same population sample. Furthermore, the HRS samples across the age range of older adults, including the oldest old. Finally, the HRS is a biennial longitudinal survey that includes utilization, disability, and mortality data, making possible future studies that examine the cross-sectional and longitudinal association of frailty with these outcomes.

Frailty models derived from different theoretical constructs identify different groups of older adults at risk for disability and other adverse outcomes. Future research is needed to compare the longitudinal outcomes of frailty for the different models. The outcomes of frailty may vary for the different models, or the models may identify different paths or trajectories to the same adverse outcomes. Rather than choosing a single model of frailty, different models with different theoretical constructs may point to different interventions to interrupt the processes resulting in frailty, with the aims of enhancing function, minimizing disability, and delaying death.

\section{ACKNOWLEDGMENTS}

The authors gratefully acknowledge Cathy Emiline-Fagan for her assistance with manuscript preparation and Yuo-Yu Lee for her assistance with data analysis. An early version of this paper was presented at the Center for Social Epidemiology and Population Health and the Robert Wood Johnson Health \& Society Scholars Program Small Grant Program Awardees Conference (University of Michigan) in May 2007.

Conflict of Interest: This work was supported by a Robert Wood Johnson Health and Society Scholars Program Small Grants Program (University of Michigan) pilot grant to Dr. Cigolle. Dr. Cigolle was supported by a Ruth L. Kirschstein National Research Service Award from the National Institute on Aging (1F32AG027649-01), the NIH-NCRR KL2 (former K12) Mentored Clinical Scholars Program at the University of Michigan, the Ann Arbor Veterans Affairs Geriatric Research, Education and Clinical Center (VA-GRECC), and the John A. Hartford Foundation Center of Excellence in Geriatrics at the University of Michigan. Dr. Blaum was supported by NIA R01 AG021493A and the Ann Arbor VA-GRECC.

Author Contributions: Dr. Cigolle and Dr. Blaum: study concept and design, analysis and interpretation of data, and preparation of the manuscript. Dr. Ofstedal: analysis and interpretation of data and preparation of the manuscript. Ms Tian: analysis and interpretation of data.

Sponsor's Role: The funding sources had no role in the design, methods, data analysis, or preparation of the manuscript.

\section{REFERENCES}

1. Ferrucci L, Guralnik JM, Studenski S et al. Designing randomized, controlled trials aimed at preventing or delaying functional decline and disability in frail, older persons: A consensus report. J Am Geriatr Soc 2004;52:625-634.

2. Levers MJ, Estabrooks CA, Ross Kerr JC. Factors contributing to frailty: Literature review. J Adv Nurs 2006;56:282-291.

3. Markle-Reid M, Browne G. Conceptualizations of frailty in relation to older adults. J Adv Nurs 2003;44:58-68.

4. Walston J, Hadley EC, Ferrucci L et al. Research agenda for frailty in older adults: Toward a better understanding of physiology and etiology: Summary from the American Geriatrics Society/National Institute on aging research conference on frailty in older adults. J Am Geriatr Soc 2006;54:991-1001.

5. Volpi E, Nazemi R, Fujita S. Muscle tissue changes with aging. Curr Opin Clin Nutr Metab Care 2004;7:405-410.

6. Roubenoff R, Harris TB. Failure to thrive, sarcopenia and functional decline in the elderly. Clin Geriatr Med 1997;13:613-622. 
7. Karlamangla AS, Singer BH, McEwen BS et al. Allostatic load as a predictor of functional decline. MacArthur Studies of Successful Aging. J Clin Epidemiol 2002;55:696-710.

8. Strawbridge WJ, Shema SJ, Balfour JL et al. Antecedents of frailty over three decades in an older cohort. J Gerontol B Psychol Sci Soc Sci 1998;53B:S9-S16.

9. Rockwood K, Andrew M, Mitnitski A. A comparison of two approaches to measuring frailty in elderly people. J Gerontol A Biol Sci Med Sci 2007;62A: $738-743$.

10. Canadian Study of Health and Aging: Study methods and prevalence of dementia. Can Med Assoc J 1994;150:899-913.

11. Rockwood K, Song X, MacKnight C et al. A global clinical measure of fitness and frailty in elderly people. Can Med Assoc J 2005;173:489-495.

12. Fried LP, Ferrucci L, Darer J et al. Untangling the concepts of disability, frailty, and comorbidity: Implications for improved targeting and care. J Gerontol A Biol Sci Med Sci 2004;59A:255-263.

13. Fried LP, Tangen CM, Walston J et al. Frailty in older adults: Evidence for a phenotype. J Gerontol A Biol Sci Med Sci 2001;56A:M146-M156.

14. Fried LP, Borhani NO, Enright P et al. The Cardiovascular Health Study: Design and rationale. Ann Epidemiol 1991;1:263-276.

15. Bandeen-Roche K, Xue QL, Ferrucci L et al. Phenotype of frailty: Characterization in the Women's Health and Aging Studies. J Gerontol A Biol Sci Med Sci 2006;61A:262-266.

16. Juster FT, Suzman R. An overview of the Health and Retirement Study. J Hum Resour 1995;30(Supplement):S7-S56.

17. Soldo BJ, Hurd MD, Rodgers WL et al. Asset and health dynamics among the oldest old: An overview of the AHEAD study. J Gerontol B Psychol Sci Soc Sci 1997;52 Spec No:1-20.

18. Orme JG, Reis J, Herz EJ. Factorial and discriminant validity of the Center for Epidemiological Studies Depression (CES-D) scale. J Clin Psychol 1986;42:2833.

19. Siscovick DS, Fried L, Mittelmark M et al. Exercise intensity and subclinical cardiovascular disease in the elderly. The Cardiovascular Health Study. Am J Epidemiol 1997;145:977-986.

20. Taylor HL, Jacobs DR Jr, Schucker B et al. A questionnaire for the assessment of leisure time physical activities. J Chronic Dis 1978;31:741-755.

21. Ainsworth BE, Haskell WL, Leon AS et al. Compendium of physical activities: Classification of energy costs of human physical activities. Med Sci Sports Exerc 1993;25:71-80.

22. Cigolle CT, Langa KM, Kabeto MU et al. Setting eligibility criteria for a carecoordination benefit. J Am Geriatr Soc 2005;53:2051-2059.

23. Cigolle CT, Langa KM, Kabeto MU et al. Geriatric conditions and disability: The Health and Retirement Study. Ann Intern Med 2007;147:156-164.

24. Folstein MF, Folstein SE, McHugh PR. "Mini-mental state". A practical method for grading the cognitive state of patients for the clinician. J Psychiatr Res 1975;12:189-198.
25. Blaum CS, Ofstedal MB, Liang J. Low cognitive performance, comorbid disease, and task-specific disability: Findings from a nationally representative survey. J Gerontol A Biol Sci Med Sci 2002;57A:M523-M531.

26. Herzog AR, Wallace RB. Measures of cognitive functioning in the AHEAD Study. J Gerontol B Psychol Sci Soc Sci 1997;52 Spec No:37-48.

27. Langa KM, Chernew ME, Kabeto MU et al. National estimates of the quantity and cost of informal caregiving for the elderly with dementia. J Gen Intern Med 2001;16:770-778.

28. Berkman LF, Breslow L. Health and Ways of Living: The Alameda County Study. New York: Oxford University Press, 1983.

29. Kulminski AM, Ukraintseva SV, Akushevich IV et al. Cumulative index of health deficiencies as a characteristic of long life. J Am Geriatr Soc 2007;55 935-940.

30. Mitnitski A, Song X, Skoog I et al. Relative fitness and frailty of elderly men and women in developed countries and their relationship with mortality. J Am Geriatr Soc 2005;53:2184-2189.

31. Woods NF, LaCroix AZ, Gray SL et al. Frailty: Emergence and consequences in women aged 65 and older in the Women's Health Initiative Observational Study. J Am Geriatr Soc 2005;53:1321-1330.

32. Ensrud KE, Ewing SK, Taylor BC et al. Comparison of 2 frailty indexes for prediction of falls, disability, fractures, and death in older women. Arch Intern Med 2008;168:382-389.

33. Ensrud KE, Ewing SK, Taylor BC et al. Frailty and risk of falls, fracture, and mortality in older women: The Study of Osteoporotic Fractures. J Gerontol A Biol Sci Med Sci 2007;62:744-751.

34. Cawthon PM, Marshall LM, Michael Y et al. Frailty in older men: Prevalence, progression, and relationship with mortality. J Am Geriatr Soc 2007;55 1216-1223.

35. Martin FC, Brighton P. Frailty: Different tools for different purposes? Age Ageing 2008;37:129-131.

36. Inouye SK, Studenski S, Tinetti ME et al. Geriatric syndromes: Clinical, research, and policy implications of a core geriatric concept. J Am Geriatr Soc 2007;55:780-791.

37. Hirsch C, Anderson ML, Newman A et al. The association of race with frailty: The Cardiovascular Health Study. Ann Epidemiol 2006;16: 545-553.

38. Studenski S, Hayes RP, Leibowitz RQ et al. Clinical global impression of change in physical frailty: Development of a measure based on clinical judgment. J Am Geriatr Soc 2004;52:1560-1566.

39. Saliba D, Elliott M, Rubenstein LZ et al. The Vulnerable Elders Survey: A too for identifying vulnerable older people in the community. J Am Geriatr Soc 2001;49:1691-1699.

40. Carey EC, Covinsky KE, Lui LY et al. Prediction of mortality in communityliving frail elderly people with long-term care needs. J Am Geriatr Soc 2008;56:68-75. 